\title{
O primeiro estudo sobre hereditariedade relacionada com as reabsorções dentárias em Ortodontia: uma análise crítica do trabalho de Newman ${ }^{21}$
}

\author{
Alberto CONSOLARO*, Maria Fernanda MARTINS-ORTIZ**, \\ Renata Bianco CONSOLARO***
}

\begin{abstract}
Resumo
$\mathrm{Na}$ literatura pertinente à relação entre reabsorções dentárias em Ortodontia e hereditariedade destacam-se quatro artigos: Newman ${ }^{21}$, Harris, Kineret, Tolley ${ }^{16}$, A1Qawasmi et al. ${ }^{1}$ e de Al-Qawasmi et $\mathrm{al}^{2}$. O trabalho de Newman ${ }^{21}$ possui o mérito do pioneirismo, mas apesar disto não conseguiu provar ou mostrar fortes evidências da relação entre reabsorções dentárias e hereditariedade. As falhas metodológicas e o pequeno número de gerações e famílias pesquisadas não permitiram afirmações conclusivas, como reconhece explicitamente o próprio autor. A principal falha está na amostra, constituída por raízes curtas sem preocupar-se com a causa das mesmas, incluindo-se raízes curtas próprias do desenvolvimento dentário. Também não houve critério definido para diagnosticar o que é uma raiz curta, foi um critério muito subjetivo. No final nota-se um pequeno número de famílias e gerações, pois foram analisados pais e filhos em apenas 17 heredogramas. Durante o trabalho não houve preocupação com a padronização do tipo de discrepâncias faciais e dentárias, do tipo de má oclusão, da morfologia radicular ou da crista óssea, do tipo de diagnóstico e plano de tratamento, nem tampouco da técnica e dos operadores dos tratamentos ortodônticos. No final considerou-se como causa de maior reabsorção de dentes com raízes curtas, após o tratamento ortodôntico, um potencial de reabsorção ao qual atribui-se um caráter genético, mas sem qualquer fundamentação nos resultados. Hoje, sabe-se que as raízes curtas quando movimentadas apresentam maior índice de reabsorção, pois concentram mais forças no ligamento periodontal, com maior possibilidade lesão na camada cementoblástica e conseqüente reabsorção radicular ${ }^{8,9,14,15,23}$.
\end{abstract}

Palavras-chave: Reabsorções dentárias. Reabsorção radicular. Genética. Hereditariedade. Movimentação ortodôntica.

* Professor Titular em Patologia da Faculdade de Odontologia de Bauru, USP.

** Mestre em Ortodontia e Doutoranda em Patologia Bucal pela FOB-USP.

*** Mestranda em Patologia Bucal da FOB-USP. 


\section{Introdução}

O trabalho de Warren G. Newman ${ }^{21}$ foi o primeiro na literatura propondo-se a estudar em famílias a relação da hereditariedade com a reabsorção dentária, com ou sem movimentação ortodôntica. Foi publicado no American Journal of Orthodontics (v.67, p.522-39) em 1975 com o título Possible etiologic factor in external root resorption ou "Possiveis fatores etiológicos na reabsorção radicular externa" e foi apresentado para a obtenção do grau de Mestre em Ciências Odontológicas na University of Washington, em Seattle, Washington, EUA. Para isto, identificou pacientes com raízes curtas diagnosticadas radiograficamente em películas periapicais, sem se preocupar se elas estavam curtas por problemas durante o desenvolvimento radicular ${ }^{9-11,18}$ ou por reabsorção dentária propriamente dita; não houve preocupação em diferenciar estes aspectos nos diagnósticos.

A literatura apresenta quatro trabalhos ${ }^{1,2,16,21}$ com a finalidade de estudar a natureza genética e hereditária das reabsorções dentárias. O trabalho de Newman ${ }^{21}$ é sempre citado como ponto de partida para os demais. Sua tradução para o português, bem como esta análise crítica, constituem uma oportunidade de aprofundamento do assunto e um resgate histórico das formas de abordagem das reabsorções dentárias na década de 1970, percebendo-se que pouco mudou nos últimos anos.

Em sua introdução Newman ${ }^{21}$ afirma: " $O o b$ jetivo deste estudo foi investigar a relação da reabsorção radicular idiopática com (1) as influências genéticas nos familiares imediatos, (2) as causas sistêmicas freqüentemente citadas na literatura, (3) o tipo de má oclusão, incluindo exame para contatos não fisiológicos durante movimentos excursivos de hipo e hiperfunção durante oclusão normal, (4) a história pregressa médica e dentária e (5) a avaliação da reabsorção radicular em pessoas submetidas a tratamento ortodôntico".

A hereditariedade das reabsorções dentárias não foi um objetivo específico do trabalho de Newman ${ }^{21}$ e o título escolhido para o trabalho foi muito coerente com seus resultados, tratando-os como possíveis fatores etiológicos sem qualquer exagero de afirmação, deixando claro que sua interpretação possuía importantes limitações metodológicas.

\section{Sobre a metodologia}

No Material e Métodos o autor relata que usou uma classificação de pacientes de acordo com o número de dentes afetados e o grau de reabsorção diagnosticado para incluí-los na amostra como assim descreveu: "Evidências radiográficas de encurtamento radicular de moderado a severo em um mínimo de três dentes em pelo menos dois quadrantes diferentes foi o primeiro critério. O dente foi considerado "severamente" encurtado quando a perda foi maior ou igual a um terço do comprimento da raiz normal (Fig. 1) e "moderadamente" encurtado se maior que $2 \mathrm{~mm}$, porém menor que um terço da raiz (Fig. 2). Dentes com uma perda de aproximadamente $2 \mathrm{~mm}$ foram considerados como "arredondados" (Fig. 3).

Este método, que pode ser considerado crítico em função da quantidade de dentes que cada paciente é portador, ou seja, 32 dentes; pois são inúmeras as oportunidades de que agentes externos atuem sobre os dentes e induzam reabsorções radiculares ou fechamento apical precoce com conseqüente encurtamento das raízes durante a odontogênese $\mathrm{e}^{10,11}$. Cada dente possui sua história pregressa, morfologia específica e relações periodontais e ósseas individuais que podem influenciar na maior ou menor reabsorção, principalmente quando submetidos ao tratamento ortodôntico. Incluir os pacientes em categorias de acordo com o número de dentes afetados permite que um dente severamente comprometido, até mesmo com seu terço cervical envolvido, possa estar presente, mas o paciente não seria selecionado, pois para incluí-lo seriam necessárias evidências radiográficas de encurtamento radicular moderado a severo em um mínimo de três dentes, em pelo menos dois quadrantes diferentes. Este foi o principal critério de inclusão na amostra. Os pacientes não enquadrados desta forma não poderiam ter relação hereditária com as reabsorções radiculares? Em casos de reabsorção em apenas um dente não haveria então fenômenos de natureza genética? 


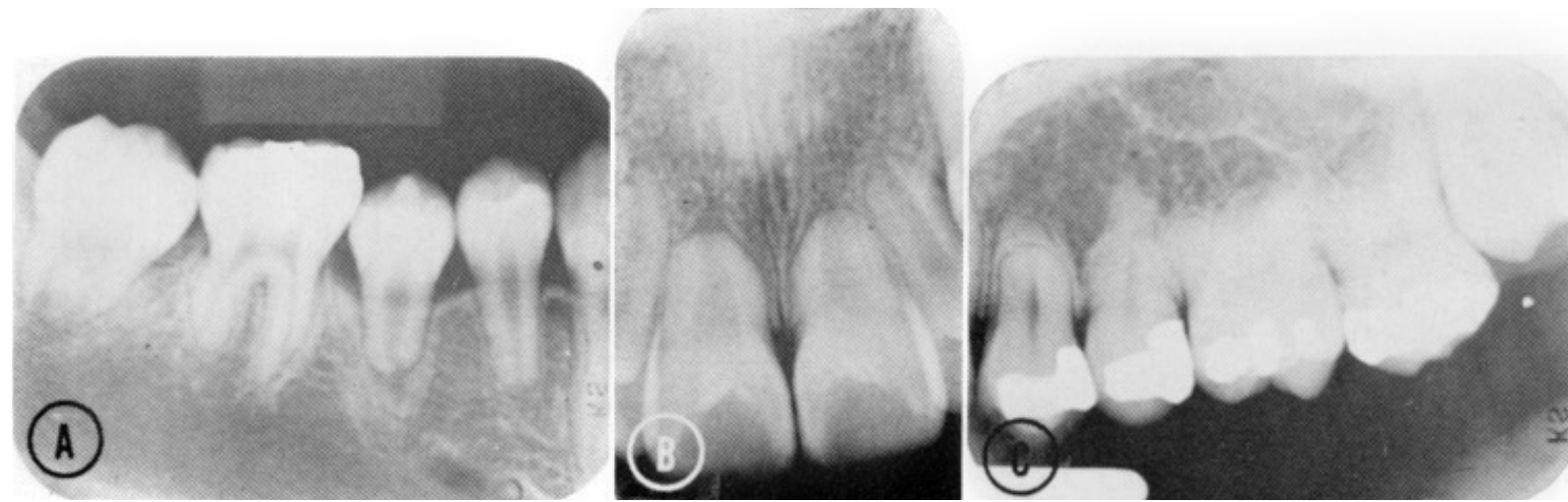

FIGURA 1 - "Encurtamento apical "severo" (dentes não tratados). A) Raízes severamente encurtadas no primeiro molar e no segundo pré-molar inferior direito; B) Raízes severamente encurtadas nos incisivos centrais superiores; C) Raizes severamente encurtadas nos pré-molares e molares superiores." 0 s dentes assim apresentados por Newman ${ }^{21}$ revelam a possibilidade destas raízes curtas estarem relacionadas com trauma oclusal, bruxismo de traumatismo dentário em função da forma radicular determinada pela reabsorção, bem como pelas alterações ósseas e periodontais. No trabalho não se identificaram nas figuras os pacientes aos quais pertenciam as radiografias e sua posição nos heredogramas apresentados.

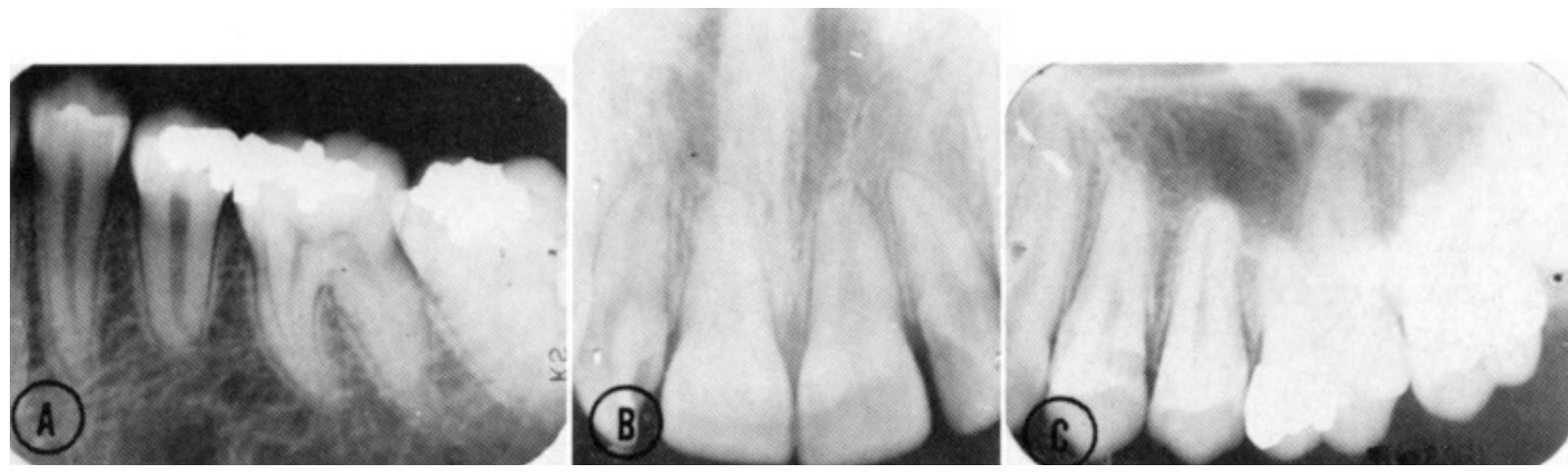

FIGURA 2 - "Encurtamento apical "moderado" (dentes não tratados). A) Raízes moderadamente encurtadas no segundo pré-molar inferior esquerdo; B) Raízes moderadamente encurtadas nos incisivos centrais superiores; C) Raízes moderadamente encurtadas no primeiro molar superior esquerdo (raízes mesio e distovestibular)." 0 s dentes assim apresentados por Newman ${ }^{21}$ revelam outras possibilidades etiológicas como na figura A onde o pré-molar pode ter fechado precocemente 0 ápice em decorrência de trauma oclusal e em B por traumatismo dentário. No trabalho não se identificaram nas figuras os pacientes aos quais pertenciam as radiografias e sua posição nos heredogramas apresentados

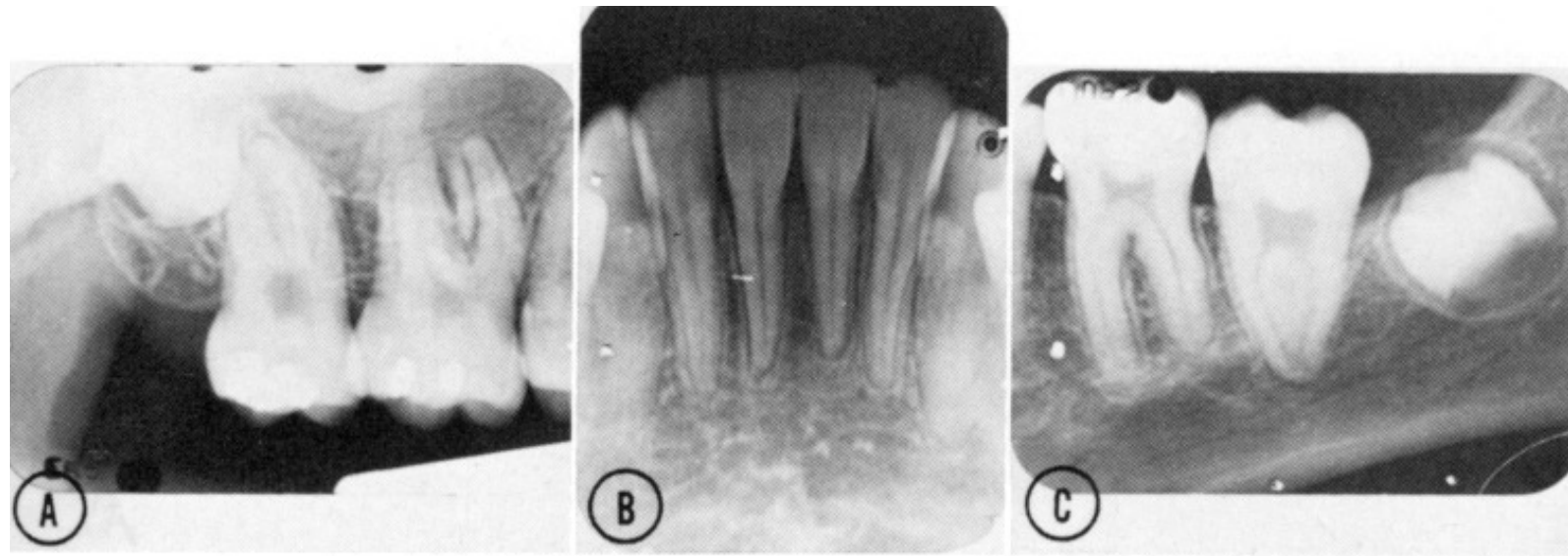

FIGURA 3 - "Ápices "arredondados" (dentes não tratados). A) Arredondamento da raiz do primeiro molar superior direito (raiz distovestibular); B) Arredondamento das raízes dos incisivos centrais inferiores; C) Arredondamento da raiz do primeiro molar inferior esquerdo (raiz distal)". Os casos apresentados podem ser questionados quanto ao diagnóstico da reabsorção, pois podem ser variações de forma e tamanho da raiz dentária, que podem ser determinadas geneticamente e transmitida hereditariamente, embora sofram influência de fatores ambientais. No trabalho não se identificaram nas figuras os pacientes aos quais pertenciam as radiografias e sua posição nos heredogramas apresentados. 
$\mathrm{Na}$ construção da amostra, o autor teve o cuidado de excluir os casos de traumatismo acidental e com trauma oclusal induzido por restaurações. Apesar destes cuidados, é reconhecido que a maior parte dos traumatismos dentários não são diagnosticados ou sequer lembrados pelo paciente, mesmo em uma anamnese minuciosa. Logo, muitos dentes incluídos podem ter sido traumatizados, mas este fato é quase impossível de ser contornado na constituição de uma amostra e deve ser relevado na discussão dos resultados.

Quanto aos critérios de interpretação radiográfica destaca-se o uso de radiografias periapicais. Mesmo assim o autor reconhece explicitamente as dificuldades encontradas no diagnóstico. O autor afirmou que: "As radiografias mostraram-se o maior problema e a angulação deficiente resultou em muitos dentes colocados em categoria questionável. Examinaram-se os filmes com extremo cuidado. Porém, sem evidência da extensão original da raiz ou qual o comprimento final, a decisão permaneceu uma questão de julgamento subjetivo".

Outro fator questionável diz respeito às múltiplas fontes de pacientes para compor a amostra: eram de vários locais e submetidos a tratamentos por vários operadores diferentes. No trabalho não houve abordagem sobre isto; os critérios de diagnósticos e tratamentos foram diferentes, mas não comentados. Apenas explicitou que "A amostra foi obtida de uma ampla variedade de fontes, incluindo os registros de Richard A. Riedel e casos de muitos ortodontistas da costa oeste dos Estados Unidos, Canadá, e México".

Para o estudo de uma situação clínica multifatorial como a reabsorção radicular, a uniformização da amostra eliminando-se o maior número das principais variáveis se faz importante, pois pode constituir um fator limitante da credibilidade dos resultados.

Das 47 pessoas que deram início ao estudo resultou-se em 37 famílias estudadas. Destas 37 famílias, 17 puderam ser avaliadas completamente - entendeu-se, nesse trabalho, como famílias completas em sua análise quando o estudo dos pais e de todos os filhos foi viabilizado. Os heredogramas estabelecidos são pequenos demais para se determinar um padrão de transmissão hereditária, o número de gerações e pessoas é muito reduzido, especialmente porque não se utilizaram técnicas de biologia molecular. O número de famílias estudadas pode ser considerado muito restrito.

\section{Sobre a observação realizada}

Uma ressalva a ser feita na leitura do trabalho refere-se aos termos incidência e prevalência. $\mathrm{O}$ autor usa indevidamente incidência no lugar de prevalência em todo texto. A incidência refere-se à freqüência de um fenômeno ou doença em uma determinada população durante um determinado período de tempo, enquanto prevalência refere-se aos níveis percentuais de comprometimento de uma população estudada, sem se preocupar com o todo da população em um período de tempo.

A prevalência de reabsorção dentária descrita para cada dente nos pacientes examinados, em nenhum momento do trabalho, foi relacionada com variáveis inerentes à técnica ortodôntica aplicada, o mesmo ocorrendo quanto à morfologia radicular e da crista óssea alveolar. Não houve padronização ou agrupamentos de dentes ou de pacientes quanto às morfologias radiculares, da crista óssea e nem mesmo quanto ao tipo de má oclusão.

\section{Quanto aos achados nas famílias e seus he- redogramas}

Qual a probabilidade de fenômenos detectados em duas pessoas em um mesmo heredograma serem achados coincidentes no contexto de uma situação tão multifatorial em relação à etiopatogenia, quanto mais se associado a tantos mediadores químicos no seu gerenciamento metabólico, celular e tecidual? Do ponto de vista clínico é muito grande a probabilidade de fatores iguais ou diferentes atuarem em duas pessoas da mesma família.

Quando três ou quatro irmãos submetidos a tratamento ortodôntico apresentam o mesmo grau de reabsorção, não existe conotação hereditária, pois estes podem possuir os mesmos tipos de discrepâncias maxilares e dentárias, o mesmo 
tipo de morfologia facial e dentária e o mesmo tipo de má oclusão, afinal são irmãos ${ }^{17,20}$. Logo, este quadro clínico levará ao mesmo tipo de tratamento, de intensidades semelhantes de forças, extensão do movimento a ser percorridos pelos dentes e o tipo de mecânica escolhida! Para que os estudos com esta natureza estejam metodologicamente adequados requer-se grupos controles, como por exemplo, um grupo em paralelo de três ou quatro indivíduos com características faciais e dentárias semelhantes ao dos quatro irmãos, empregando-se nestes, o mesmo tipo de tratamento ortodôntico. Infelizmente não encontramos trabalhos sobre reabsorção dentária e hereditariedade com esta preocupação metodológica de se ter um grupo controle sem parentesco, mas com as mesmas variáveis clínicas.

Os resultados dos heredogramas apresentados não permitem uma avaliação segura de hereditariedade na amostra apresentada. O número de famílias e gerações analisadas é pequeno, principalmente se considerarmos que o objeto de estudo, as reabsorções dentárias, representa um evento com múltiplas causas e fatores influenciando seu estabelecimento inicial e sua evolução. Além disto, nas 17 famílias estudadas a distribuição do possível padrão de transmissão hereditária foi muito heterogênea, reduzindo a amostra a pequenos subgrupos de 3 a 6 famílias cada.

Em sua discussão, Newman ${ }^{21}$ afirma: "Encontrou-se uma amostra de pessoas muito maior com reabsorção radicular "idiopática" moderada e severa múltipla do que a originalmente esperada, embora nada se possa concluir sobre uma prevalência específica."

\section{Sobre os dados de reabsorção após o trata- mento ortodôntico}

Os resultados percentuais de Newman ${ }^{21}$ sobre a ocorrência de reabsorções dentárias após o tratamento ortodôntico, especialmente dos dentes que anteriormente apresentavam raízes encurtadas, confirmaram as observações de Furquim $^{14,15}$, Consolaro et al. ${ }^{8}$ e Velloso et $\mathrm{al}^{23}$, além de outros ${ }^{7}$, revelando que quanto mais curta a raiz, maior a previsibilidade de reabsorção dentária no prognóstico de um tratamento orto- dôntico. Oppenheim ${ }^{22}$ em 1936 foi o primeiro a postular que a forma da raiz era importante para predispor o dente à reabsorção radicular em Ortodontia. Isto por sua vez não indica sinais de hereditariedade, mas sim que a morfologia e a proporção coroa-raiz interferem na maior ou menor ocorrência de reabsorção de um determinado dente durante o tratamento ortodôntico.

A raiz curta, assim como a morfologia radicular triangular associada ou não a uma crista óssea retangular, quando submetida a uma determinada força ortodôntica seria como a pela clara exposta ao sol. A probabilidade de a pele clara ficar avermelhada, com sardas, manchas senis e apresentar câncer de pele é muito maior do que em pessoas de pele escura. Entretanto, nunca se ouviu falar que pessoas de pele clara apresentam uma predisposição genética individual ou hereditária ao câncer de pele. Apenas que peles mais escuras apresentam uma pigmentação maior protegendo melhor o material genético contido no núcleo. O genoma de indivíduos com diferentes tonalidades de pele é o mesmo, mudando um ou outro alelo dominante ou recessivo alterando o fenótipo, ou seja a manifestação clínica de determinada característica.

Assim é a raiz curta, triangular, dilacerada ou em forma de pipeta. Simplesmente estas características morfológicas favorecem uma maior concentração da força ortodôntica provocando a morte de cementoblastos e reabsorção ${ }^{9,14,15 \text {, }}$ ${ }^{23}$. Mas a força ortodôntica constitui um agente local e externo controlável, que pode ser mais bem dimensionado quando do diagnóstico prévio destas especificidades morfológicas. Raízes mais rombóides ou retangulares distribuem melhor as forças ortodônticas no ligamento periodontal, ao longo da superfície radicular apical, provocando menos hipóxia e estresse, apenas o suficiente para estimular a remodelação do osso alveolar, permitindo o movimento dentário, sem lesar a camada cementoblástica e sem provocar reabsorções ${ }^{9,14,15,23}$.

\section{Quanto à variabilidade na prevalência de re- absorções dentárias}

Após 1975, inúmeros outros trabalhos sobre prevalência de reabsorções em Ortodontia fo- 
ram publicados. Nas amostras relatadas os índices percentuais são compativeis com os apresentados pelo autor neste trabalho. Esta compatibilidade de resultados fica reforçada mais ainda se considerarmos as morfologias radiculares, tais como as raízes curtas, agora reconhecidas como fatores importantes na previsibilidade e na freqüência de reabsorções em Ortodontia ${ }^{9}$. A variabilidade percentual encontrada nas casuísticas relaciona-se também à heterogeneidade das amostras utilizadas, sem padronização técnicas, de características diagnósticas ou morfológicas.

\section{Quanto à prevalência da reabsorção dentária e o gênero}

A maior prevalência de reabsorções dentárias em mulheres observadas por Newman ${ }^{21}$ não se confirmou nos inúmeros outros trabalhos em que amostras mais equilibradas e minuciosamente analisadas foram comparadas quanto ao gênero. Um levantamento completo e minucioso da literatura atual pertinente revela que o gênero não interfere no menor ou maior índice de reabsorção dentária durante o tratamento ortodôntico. Beck e Harris ${ }^{4}$, em 1994, declararam: "Nenhuma diferença foi encontrada entre os gêneros. Intuitivamente, as consideráveis diferenças nos níveis de vários hormônios entre homens e mulheres fez, no passado, sugerir que existisse diferença na susceptibilidade às reabsorções dentárias. De fato, esta diferença não é consistente".

\section{Quanto à susceptibilidade dos dentes às re- absorções}

A maior prevalência de reabsorção em um determinado tipo de dente deve ser criteriosamente avaliada, pois esta reabsorção pode decorrer de uma maior exigência periodontal, quanto ao tipo de movimento e a intensidade de força aplicada sobre ele e não em função da susceptibilidade ou predisposição estrutural hipoteticamente herdada geneticamente. $\mathrm{O}$ termo susceptibilidade como sinônimo de prevalência maior pode possuir uma conotação equivocada. Os dentes com maior prevalência geralmente são os mais exigidos e movimentados durante o tratamento ortodôntico, bem como os que apre- sentam formas radiculares que tendem a concentrar mais força no ápice, conseqüentemente, lesando com maior probabilidade a camada celular cementoblástica que protege a raiz da reabsorção.

Apesar da forma radicular ser geneticamente transmitida pelos pais, ela sofre uma forte influência de fatores ambientais como densidade óssea, obstáculos encontrados durante a trajetória eruptiva e força oclusal durante a rizogênese em sua fase final 10,11,18. Apesar de geneticamente influenciada, a forma da raiz não pode ser considerada como hereditariamente determinada de forma exclusiva, menos ainda as reabsorções dentárias, mesmo que mais prevalentes em um determinado dente em questão.

Quanto maior a prevalência de reabsorção em determinados dentes, deve-se procurar as causas da reabsorção visualizando nas radiografias periapicais, as características morfológicas dentárias, do tipo de força e movimento aplicados. Nos incisivos e caninos, em função da sua posição anterior e forma radicular, em radiografias periapicais, a reabsorção é precocemente detectada, enquanto que nos pré-molares e molares, devido à morfologia e local de ocorrência das reabsorções, em geral nas suas faces laterais, dificilmente são visualizadas, a não ser quando avançadas, como ocorre na utilização de aparelhos extrabucais e do tipo pêndulo, por exemplo.

As várias prevalências de reabsorções encontradas e relatadas pelo autor em casuísticas diferentes podem ser explicadas pela falta de padronização da amostra. Não se deve analisar um fenômeno como a reabsorção dentária abarcando, no mesmo grupo de pacientes, os mais variados tipos de má oclusão, de morfologia facial e dentária, vários tipos de tratamentos ortodônticos e ainda com vários operadores diferentes, em tempos diferentes.

Não se apresenta como preocupação dos autores a homogeneização da amostra durante a sua seleção na quase totalidade dos trabalhos propondo-se a estudar a freqüência e as causas das reabsorções, especialmente nas relacionadas com o tratamento ortodôntico.

A homogeneização de uma amostra implica 
na possibilidade de reduzir a quantidade de espécimes; por outro lado se ela não for possivel, que se tente o máximo de padronização e aumente consideravelmente o número de espécimes.

\section{Sobre o diagnóstico radiográfico}

Uma limitação devidamente ressaltada pelo autor em vários pontos do trabalho refere-se ao método radiográfico para a mensuração da reabsorção. Os pacientes foram classificados em quatro categorias:

0 = nenhuma reabsorção ou encurtamento;

1 = encurtamento radicular questionável;

2 = encurtamento radicular definido, mas não severo;

$3=$ encurtamento severo.

Em seu texto, Newman"21 afirmou: "Nesses critérios incluiu-se a raiz reabsorvida tendo um forame apical visivel, mas sem um término afilado aparente do canal como se vê normalmente. Na maioria dos casos, não foram feitas tentativas para determinar a natureza do processo, se era do desenvolvimento ou de natureza reabsortiva; em vários dentes nenhum critério conclusivo pode ser usado". O autor considerou esta limitação muito importante e reconheceu a subjetividade do método aplicado. Apesar da utilização de radiografias periapicais não se mediu objetivamente a perda de comprimento da raiz, pois se alegou que não havia como obter o tamanho original da raiz, na maioria dos casos, por limitações técnicas na obtenção das radiografias como, por exemplo, na padronização da angulação.

$\mathrm{Na}$ discussão de sua amostra o autor afirma: "Na presente investigação a amostra foi especificamente selecionada para estudar-se a etiologia de múltiplos dentes com raízes curtas. $O$ exame radiográfico de dentes individuais foi difícil e a classificação final poderia ser descrita como muito conservadora". Em outro parágrafo assim se expressou: "O principal problema na seleção da amostra foi a interpretação radiográfica. A qualidade das radiografias periapicais disponíveis variaram consideravelmente e todo esforço foi feito para obter-se várias tomadas radiográficas do mesmo dente. Como mencionado previamente, a comparação de dentes adjacentes para determinação da proporção coroa-raiz foi realizada por comparação com outras películas obtidas."

\section{Sobre a discussão do trabalho}

Neste trabalho, em vários pontos do texto, o autor ressalta que o seu objeto de análise foram os dentes com raízes curtas ou encurtadas, sem necessariamente distinguir se o foram por distúrbios do desenvolvimento dentário ou por reabsorção radicular. Assim o fez por considerar difícil a distinção. Deve-se relevar que o comprimento de uma raiz dentária condiciona-se a fatores genéticos, mas fortemente influenciado por fatores ambientais, como revelou Kovacs ${ }^{18}$. Logo, os dados relacionando hereditariedade ao comprimento das raízes não necessariamente associam-se reabsorção dentária. Muitos dos casos considerados encurtados podem ser conseqüência de fatores ambientais interferindo no desenvolvimento normal das raízes dentárias.

Um dado relevante na discussão do trabatho refere-se à possibilidade do ápice fechar-se precocemente, ainda durante a rizogênese, em função da carga oclusal excessiva, o que denota uma corroboração com o raciocínio de que fatores ambientais podem influenciar significativamente no comprimento da raiz, conforme revisado por Consolaro, Martins-Ortiz e Veloso $^{10}$. Para indicar o papel das forças oclusais excessivas no encurtamento das raízes o autor cita o trabalho de Ando et $\mathrm{al}^{3}$.

\section{Relação da mordida aberta com as reabsor- ções dentárias}

As reabsorções dentárias estão relacionadas com a maior extensão do movimento ortodôntico, que pode estar relacionada com a severidade do mal posicionamento dentário. Os pacientes com mordida aberta anterior apresentam maior possibilidade de reabsorção dentária pré-tratamento, provocada por traumatismos dentários, por hábitos e vícios, incluindo-se a pressão lingual e, ao mesmo tempo, apresentam uma tábua óssea vestibular mais fina. Quando da maior reabsorção encontrada nos dentes posteriores, nos casos de mordida aberta anterior, deve-se lembrar que estes dentes suportam toda a carga 
oclusal que deveria ser distribuída entre todos os elementos dentários. Ademais, grande parte, senão a maioria das correções ortodônticas de mordidas abertas anteriores, é corrigida com extração e retração anterior, exigindo tanto dos dentes anteriores quanto da unidade de ancoragem. A história anterior de traumatismos e os movimentos de inclinação são fatores importantes na compreensão das reabsorções dentárias durante o tratamento ortodôntico. $\mathrm{O}$ autor não explorou nenhum destes aspectos para explicar seus dados.

\section{Sobre a relação entre genética, hereditarie- dade e reabsorção dentária}

$\mathrm{Na}$ discussão sobre os aspectos genéticos das raízes encurtadas, o autor ressalta muito bem que o tamanho da amostra é muito pequeno, mas ao final deste tópico procura explicar a heterogeneidade dos resultados como resultante da heterogeneidade dos pacientes que compunham sua amostra. Mesmos em estudos envolvendo biologia molecular, com base nos métodos atuais, os estudos genéticos requerem uma amostra significante numericamente. Em apenas 17 famílias e considerando as múltiplas causas e fatores envolvidos na etiopatogenia das reabsorções dentárias, qualquer afirmação com caráter ou roupagem de conclusão apresenta-se fragilizada. $\mathrm{O}$ autor do referido trabalho repetidamente reconhece as limitações de suas conclusões quanto à hereditariedade e sua relação com as reabsorções dentárias.

$\mathrm{O}$ autor afirma que nenhuma conclusão genética definitiva pode ser extraída do seu trabalho, mas em alguns pontos procura valorizar o papel da genética na etiopatogenia das reabsorções dentárias, porém sem fundamentar-se em dados revelados pelo trabalho. $\mathrm{Na}$ discussão dos resultados destaca-se uma de suas afirmações: "Os dados dos heredogramas, tanto de famílias consideradas completas quanto incompletas, sugerem um componente genético para as raízes encurtadas. Porém, nenhuma análise de segregação específica poderia ser executada por causa da pequena amostra de famílias completas analisadas e da heterogeneidade genética aparente destes estudos familiares. Na proposta desta discussão, considerou-se somente família examinada por completo. Heredogramas com um ou mais irmãos afetados e apenas um dos pais afetado sugere transmissão autossômica dominante; seis famílias foram compativeis com este padrão, embora o tamanho pequeno da amostra não permita conclusões definitivas".

Em vários pontos do texto do trabalho, o autor refere-se a raízes encurtadas ou curtas, pois não conseguiu distinguir se eram curtas decorrentes do fechamento precoce do ápice durante o desenvolvimento dentário ou se eram verdadeiramente reabsorções radiculares. Lind et al. ${ }^{19}$ descrevem as raízes curtas como parte integrante de um quadro clínico denominado Síndrome das Raízes Curtas e para ele, as raízes curtas podem ser herdadas. É provável que alguns casos familiares de raízes "encurtadas" da amostra de Newman ${ }^{21}$ sejam distúrbios do desenvolvimento dentário e não casos de reabsorções radiculares.

\section{Sobre os fatores sistêmicos e as reabsorções dentárias}

Newman ${ }^{21}$ menciona e critica severamente os trabalhos de Becks $^{5-7}$ e seus colaboradores que entre 1936 e 1942 publicaram artigos que estudaram a relação entre reabsorção dentária e fatores sistêmicos. Para Newman ${ }^{21}$, Becks $^{5-7}$ e seus colaboradores atribuíram os fatores sistêmicos como causas das reabsorções dentárias, especialmente as endocrinopatias. Entretanto parece-nos justos resgatar estes trabalhos ${ }^{5-7}$, nos quais em nenhum momento enfatizaram ou relacionaram os distúrbios sistêmicos como causas das reabsorções dentárias. Para justificar este questionamento às afirmações de $\mathrm{Newman}^{21}$ sobre Becks e colaboradores ${ }^{5-7}$ resgatamos algumas frases dos seus trabalhos originais que apresentamos a seguir:

Becks" (1936): "esses dados não justificam afirmar que as várias doenças endócrinas estudadas produziram as reabsorções dentárias e sim que com elas coexistem".

Becks $^{6}$ (1939): "as reabsorções dentárias apenas coexistem com os distúrbios sistêmicos e não há provas cientificas que resultem dos mesmos. Os distúrbios sistêmicos podem ser eliminados 
como fatores de risco na prática ortodôntica".

O trabalho de Becks ${ }^{6}$ de 1939 possui 14 páginas com vários gráficos e tabelas. Apesar de muito criterioso e esclarecedor quanto ao não envolvimento das endocrinopatias como causas das reabsorções dentárias com e sem tratamento ortodôntico, é comum encontrar referências a este artigo como prova de relação entre endocrinopatias e reabsorção dentária em vários textos de artigos e livros. Isto pode ser atribuído a falhas de interpretação na leitura do trabalho, pois o título até pode, em uma leitura rápida, sugerir esta relação: "Orthodontic prognosis evaluation of routine dentomedical examinations to determine: good and poor risks".

$\mathrm{O}$ mais intrigante é que o próprio Newman ${ }^{21}$ critica os trabalhos de Becks $s^{5-7}$, que dominaram o pensamento durante décadas a respeito de eventuais fatores sistêmicos e hormonais para as reabsorções, considerando-os dogmáticos e pobres. Ele próprio revela: "Os ortodontistas geralmente estavam muito irritados pelo fato de que poderiam ser os responsáveis pela reabsorção radicular; este estigma provavelmente envolveria várias conseqüências legais e profissionais desagradáveis. Qualquer investigador respeitado que conduzisse um estudo razoavelmente bem organizado e que levasse a encontrar qualquer coisa diferente das mecânicas de tratamento ortodôntico como a causa de reabsorção radicular, seria aclamado".

Em 1942, Becks e Cowden ${ }^{7}$ publicaram um trabalho sobre reabsorções dentárias e suas relações com problemas endócrinos e afirmaram que seus métodos e resultados não permitiam concluir sobre as endocrinopatias como causas de reabsorções dentárias. Para eles esta relação requer provas científicas e confirmações laboratoriais. Entre as endocrinopatias possivelmente relacionadas com as reabsorções, os autores valorizaram o hipotireoidismo. Em 2002, Francischone $^{12,13}$, em sua tese de doutorado, analisou 81 pacientes endocrinopatas distribuídos em grupos de 10 pacientes, em nove situações clínicas endócrinas diferentes. Após uma análise minuciosa de todas as raízes dentárias e das análises bioquímicas, concluiu que as reabsorções dentárias não fazem parte do quadro de mani- festações clínicas e radiográficas das endocrinopatias.

No mesmo ano, Furquim ${ }^{14,15}$, em outra tese de doutorado, ao analisar o perfil endocrinológico de pacientes ortodônticos com e sem reabsorções dentárias, não encontrou qualquer diferença nos níveis hormonais e bioquímicos dos pacientes. Ao comparar os três grupos de 70 pacientes cada um, sendo um grupo sem tratamento ortodôntico, um segundo grupo com tratamento ortodôntico e sem reabsorções dentárias e um terceiro grupo, com tratamento ortodôntico e com reabsorções dentárias, o autor detectou que a diferença maior nos três grupos experimentais estava no tipo de morfologia radicular e da crista óssea alveolar. As raízes triangulares e as cristas ósseas retangulares estavam mais prevalentes no grupo com reabsorções dentárias.

Um aspecto muito interessante no texto da discussão de Newman ${ }^{21}$, refere-se à supervalorização dos fatores sistêmicos por parte dos ortodontistas na época de Becks ${ }^{5-7}$ e colaboradores, ou seja, nas décadas de 1930 e 1940. Para Newman ${ }^{21}$, essa supervalorização ocorreu para atenuar as questões iatrogênicas das reabsorções na prática ortodôntica, incluindo-se as questões legais.

No final, o autor apresenta como uma de suas conclusões: "Acreditou-se anteriormente que fatores sistêmicos fossem de significado etiológico principal na reabsorção radicular "idiopática", mas nenhuma das informações colhidas nesta investigação substanciou qualquer relação entre os dois fatores. Testes sangüíneos não especificamente voltados para endocrinopatias, exceto para condições tireoidianas, não indicaram nenhum problema endócrino ou metabólico na amostra. A avaliação de radiografias de mão e punho, aparência física e histórias dentária e médica não foram essencialmente contributivas. Hipo e hipertireoidismo, especificamente, foram inexistentes".

Nos dias de hoje procura-se supervalorizar as questões genéticas e hereditárias das reabsorções, também em parte como forma de atenuar a sua natureza iatrogênica e suas implicações profissionais. No lugar de procurar causas 
evasivas ou "bodes expiatórios" a Ortodontia será mais científica se difundir o conhecimento de que as reabsorções dentárias são parte das conseqüências naturais do tratamento ortodôntico, até mesmo inevitáveis em muitos casos. Deve-se encarar as reabsorções dentárias como um custo biológico do tratamento ortodôntico para a obtenção de uma oclusão saudável e uma estética favorável. Este aspecto deveria ser amplamente informado ao paciente e à sociedade.

A freqüência de reabsorções encontradas nas variadas casuísticas ortodônticas pode ser amplamente questionada, pois são muito variadas em seus números. São raríssimos os estudos com padronização da amostra no que concerne ao tipo de má oclusão, diagnóstico inicial, discrepâncias esqueléticas e dentárias, protocolo de tratamento, técnica ortodôntica utilizada, padronização da técnica e dos operadores, detecção de história de traumatismos e muitos outros fatores que podem influenciar no índice de reabsorção. Esta falta de padronização, não oferece um índice seguro da freqüência das reabsorções em tratamentos ortodônticos.

A variabilidade dos números em decorrência da falta de padronização da amostra, da técnica e dos operadores faz com que alguns atribuam esta variabilidade à predisposição genética ou hereditária de uma ou outra população. Antes de pensarmos nos aspectos genéticos e hereditários das reabsorções dentárias, devemos conhecer os múltiplos fatores etiológicos envolvidos e padronizar as amostras estudadas.

$\mathrm{Na}$ discussão de seus resultados Newman ${ }^{21}$ acrescenta: "O tamanho da amostra neste estudo e as restrições impostas certamente acrescentaram valor na determinação da etiologia da "reabsorção radicular idiopática". Podemos ficar extasiados pela riqueza de conjecturas, teorias e suposições encontradas na literatura para a etiologia da reabsorção dentária. Na maioria dos estudos o tamanho da amostra consiste em um ou dois pacientes. Em estudos avaliando amostras maiores, uma população normal compondo a amostra apresentou reabsorções suaves. Pouco se pode concluir sobre a sua etiologia, apenas com algumas exceções."

\section{Potencial de reabsorção: uma característica hereditária?}

Newman ${ }^{21}$ afirmou que as raízes curtas podem ser resultantes de distúrbios de desenvolvimento dentário ou de reabsorções radiculares. Analisando sua amostra, afirma não ser possível tecer considerações conclusivas sobre a hereditariedade. Entretanto, ao detectar que as raízes encurtadas apresentavam reabsorções mais severas após o tratamento ortodôntico procurou responsabilizar este aumento a um possivel potencial de reabsorção que seria determinado geneticamente. Na literatura atual, parece claro que raízes mais curtas concentram mais forças nas estruturas periodontais, com maior probabilidade de lesar os tecidos periodontais, especialmente a camada cementoblástica, que protege a raiz da reabsorção ${ }^{8,9,14,15,23}$. Em síntese, raízes curtas apresentam maior índice de reabsorção dentária após o tratamento ortodôntico, se não forem poupadas quanto à intensidade de forças e tipos de movimentos aplicados. Isto decorre de fatores locais inerentes ao tratamento ortodôntico, e não devido a questões genéticas. No trabalho de Newman ${ }^{21}$, não há dados que suportem minimamente afirmações sobre a natureza genética ou hereditária das reabsorções ou a sua predisposição individual ou dentária.

O autor atribuiu ao fator genético a determinação de um potencial de reabsorção; este potencial de reabsorção, no seu entendimento, relaciona-se às raízes curtas "idiopáticas". O seu raciocínio consiste em afirmar que as raízes quando são curtas decorrem de alterações genéticas; se as raízes curtas submetidas a tratamento ortodôntico são mais susceptíveis a reabsorções durante o tratamento ortodôntico, a reabsorção dentária apresentaria uma conotação genética. Os dados apresentados em seu trabalho, em nenhuma hipótese permitem tais inferências ou conclusões.

Para finalizar a análise crítica do trabalho de Newman ${ }^{21}$ reservamos uma última observação. Durante todo o trabalho o autor refere-se a dentes com raízes curtas ou encurtadas, sendo que seu diagnóstico se baseou na morfologia radiográfica apresentada nas películas periapicais. Este método não nos parece preciso. Em traba- 
lhos de Francischone ${ }^{12,13}$, Furquim ${ }^{14,15}$, Veloso et al. ${ }^{23}$ ficou estabelecido que raízes curtas correspondem aos dentes cuja proporção raiz-coroa é igual a 1,6 . Esta proporção permite uma precisão maior, pois no trabalho analisado de Newman ${ }^{21}$ permanece a pergunta: raiz curta é apenas aquela que apresenta término abrupto do ápice ou a que possui uma proporção coroa-raiz diminuída? Parece lógico que o índice proporcional é mais preciso e promove um diagnóstico padronizado; provavelmente muitos pacientes examinados por Newman ${ }^{21}$ tinham raízes curtas, mas não foram incluídos ou computados como tal e isto pode também ter influenciado no resultado final do trabalho.

\section{Consideração final}

Após uma análise criteriosa do trabalho de Newman ${ }^{21}$ podemos afirmar: trata-se de um artigo abrangente no qual se procura principalmente avaliar o papel do trauma oclusal, dos fatores sistêmicos e da hereditariedade na etiopatogenia das reabsorções dentárias, voltando-se especialmente para as relacionadas com o movimento ortodôntico. Dentro do possível, e do conhecimento da época, o trabalho foi criterioso, mas apresentou algumas falhas metodológicas. A principal falha na análise foi na escolha dos dentes com raízes curtas, sem importar-se se eram devidos a reabsorções ou a distúrbios do desenvolvimento radicular e submetê-los a tratamento ortodôntico, ao mesmo tempo não utilizando um método quantitativo e não definindo o que foi considerado como raiz curta. Em ciência os trabalhos devem utilizar metodologias reprodutíveis por outros grupos de trabalho em outros locais e, neste caso, isto não é possível.

Quanto à relação do trauma oclusal com as raízes curtas, seus dados não revelaram interferência, mas considerando os fatores sistêmicos ele não conseguiu encontrar nenhuma influência na etiopatogenia das reabsorções dentárias em sua amostra.

A limitação metodológica por si só não permitiria relacionar reabsorções dentárias e hereditariedade com os dados apresentados, mas mesmo que não se relevasse esta limitação metodológica, o número pequeno de famílias e ge- rações estudadas também não permitiria estabelecer esta relação. Deve-se ressaltar que o autor reconheceu e explicitou estas limitações na interpretação dos resultados. Newman ${ }^{21}$ percebeu em suas análises que as raízes curtas tinham maior reabsorção apical após o tratamento ortodôntico e, ao nosso ver, equivocou-se quando interpretou isto como um potencial de reabsorção de natureza hereditária. Hoje, sabe-se que raízes curtas concentram as forças e aumentam a chance de dano aos tecidos periodontais com morte de cementoblastos e conseqüente reabsorção radicular ${ }^{8,9,14,15,23}$.

$\mathrm{O}$ profissional que ler rapidamente o artigo de Newman ${ }^{21}$ pode ter, equivocadamente, a nítida impressão que o autor conseguiu estabelecer uma relação entre a hereditariedade e as reabsorções dentárias, mas ele mesmo, ao longo do trabalho explicita que esta relação não deve ser realizada com base nos seus dados. Por fim, muitos daqueles que afirmam que a hereditariedade e reabsorções dentárias estão relacionadas, e citam este trabalho como suporte, provavelmente não o leram minuciosamente por inteiro, em todas as suas nuances. 


\title{
The first study about heredity related to dental resorptions in Orthodontics: a critical analysis of Newman's work ${ }^{21}$
}

\begin{abstract}
Four articles stand out when analyzing literature concerning root resorption in Orthodontics and heritability: Newman ${ }^{21}$, Harris, Kineret, Tolley ${ }^{16}$ and Al-Qawasmi et al. ${ }^{1}$ e Al-Qawasmi et al. ${ }^{2}$. Although Newman's study ${ }^{21}$ was pioneer on the matter, it could not prove or show strong evidence relation between dental resorption and heritability. The author acknowledges himself that there were methodological flaws. Its main problem was that short roots were included in the sample regardless of the cause inclusive those naturally short. Moreover, an unspecific and subjective criterion was used to diagnose short roots. Finally, the small number of families and generations studied does not permit to affirm conclusively. Parents and children were analyzed in only 17 pedigrees. There was no preoccupation standardizing malocclusions, facial types, root and alveolar crest morphology, diagnosis, treatment plan, used technique nor professionals involved. In the conclusions a genetic potential was considered the main cause for root resorption on short roots, not based on any evidence provide by the results. Recently, it has been shown that short roots are more likely to concentrate force on the periodontal ligament with a higher possibility of damaging the cementoblastic layer and therefore present more root resorption.
\end{abstract}

Key words: Root resorption. Dental resorption. Genetic predisposition. Heritability. Orthodontic movement. Induced tooth movement.

\section{REFERÊNCIAS}

1. AL-QAWASMI, R. A. et al. Genetic predisposition to external apical root resorption. Am J Orthod Dentofacial Orthop, St. Louis, v. 123, no. 3, p. 242-252, Mar. 2003.

2. AL-QAWASMI et al. Genetic predisposition to external apical root resorption in orthodontic patients: linkage of chromosome-18 marker. J Dent Res, Chicago, v. 82, no. 5, p. 356-360, Aug 2003.

3. ANDO, S. et al. Studies on the consecutive survey of succedaneous and permanent dentition in the Japanese children. J Nikon Univ Sch Dent, [S.I.], v. 9, p. 67-82, 1967

4. BECK, B. W.; HARRIS, E. F. Apical root resorption in orthodontically treated subjects: analysys of edgewise and light wire mechanics. Am J Orthod Dentofacial Orthop, St. Louis, v. 105, no. 4, p. 350-361, Apr. 1994.

5. BECKS, H. Root resorption and their relation to pathologic bone formation: part I. statistical data and roentinogenographic aspect. Int J Orthod Oral Surg, Copenhagenv. 22, p. 445-482, 1936.

6. BECKS, H. Orthodontic prognosis: evaluation of routine dentomedical examination to determine "good and poor risks". Am J Orthod Oral Surg, St. Louis, v. 25, p. 610-624, July 1939.

7. BECKS, H.; COWDEN, R. C. Root resorption and their relation to pathologic bone formation: part II. Classification, degrees, prognosis and frequency. Am J Orthod Oral Surg, v. 28, p. 513-536, Sept. 1942.

8. CONSOLARO, A. et al. Partial anodontia: form and size of remaining teeth and orthodontics implications. In: ANNUAL SESSION OF THE AMERICAN ASSOCIATION OF ORTHODONTICS, 101., 2001, Toronto. Anais... Toronto: American Association of Orthodontics, 2001. p. 84.

9. CONSOLARO, A. Reabsorções dentárias nas especialidades clínicas. Maringá: Dental Press International, 2002.

10. CONSOLARO, A.; MARTINS-ORTIZ, M. F.; VELLOSO, T. R. G. Dentes com rizogênese incompleta e movimento ortodôntico: bases biológicas. R Dental Press Ortodon Ortop Facial, Maringá, v. 6, n. 2, p. 25-30, mar./abr. 2001.

11. ENNES, J. P.; MARTINS - ORTIZ, M. F.; CONSOLARO, A. Incomplete root formaion: morfology and implications in orthodontics In: ANNUAL SESSION OF THE AMERICAN ASSOCIATION OF ORTHODONTICS, 101., 2001, Toronto. Anais... Toronto: American Association of Orthodontics, 2001. v. 1. p. $80-80$

12. FRANCISCHONE, T. R. C. G. Reabsorção dentária: determinação de sua freqüência em pacientes com endocrinopatias. 2002. 125 f. Tese (Doutorado em Odontologia)-Faculdade de Odontologia de Bauru, Universidade de São Paulo, Bauru, 2002. 
13. FRANCISCHONE, R. T. G; FUROUIM, L. Z : CONSOLARO A. Reabsorção dentária: determinação de sua freqüência em pacientes com endocrinopatias. In: REUNIÃO ANUAL SBPQO 19., 2002. Águas de Lindóia. Pesquisa Odontológica Brasileira: Brazilian Oral Research. São Paulo: Universidade de São Paulo, 2002. v.16.

14. FURQUIM, F. Z.; MARTINS - ORTIZ, M. F., CONSOLARO, A FRANCISCHONE, T. R. G.C. Perfil endocrinológico de pacientes ortodônticos com e sem reabsorções dentárias: correlação com a morfologia radicular In: REUNIÃO ANUAL SBPQO, 19., 2002, Águas de Lindóia. Pesquisa Odontológica Brasileira: Brazilian Oral Research. São Paulo: Universidade de São Paulo, 2002. v. 16

15. FURQUIM, L. Z. Perfil endocrinológico de pacientes ortodônticos com e sem reabsorções dentárias: correlação com a morfologia radicular e da crista óssea alveolar. 2002. $122 \mathrm{f}$ Tese (Doutorado em Odontologia)- Faculdade de Odontologia de Bauru, Universidade de São Paulo, Bauru, 2002

16. HARRIS, E. F.; KINERET, S. E.; TOLLEY, E. A. A heritable component for external apical root resorption in patients treated orthodontically. Am J Orthod Dentofacial Orthop, St. Louis, v. 111, p. 301-319, 1997
17. HU, J. R.: NAKASHIMA, A. TAKAHAMA, Y. Familial similarity in dental arch form and tooth position. J Craniofac Genet Dev Biol, Copenhagen, v. 12, p. 33-40, 1992.

18. KOVACS, I. Contribution to the ontogenetic morphology of roots of human teeth. J Dent Res, Chicago, v. 46, p. 865-874, 1967

19. LIND, V. Short root anomaly. Scand J Dent Res, Copenhagen, v. 80 , no. 2 , p. $85-93,1972$

20. NAKASHIMA, A. et al. Hereditary factor in the craniofacial morphology of Angle's Class II and Class III malocclusions. Am J Orthod, St. Louis, v. 82, no. 2, 250-256, 1982

21. NEWMAN, W. G. Possible etiologic factor in external root resorption. Am J Orthod, St. Louis, v. 67, p. 522-539, 1975.

22. OPPENHEIM, A. Biologic orthodontic therapy and reality. Angle Orthod, Appleton, v. 6, p. 153-183, 1936.

23. VELLOSO, T. R. G. et al. Anodontia Parcial: forma e tamanho dos dentes remanescentes e prováveis implicações clínicas In: REUNIÃO ANUAL DA SOCIEDADE BRASILEIRA DE PESQUISA ODONTOLÓGICA - SBPQO, 18., 2001. Águas de Lindóia. Pesquisa Odontológica Brasileira: Brazilian Oral Research. São Paulo: Universidade de São Paulo, 2001. v. 15.

Endereço para correspondência

Prof. Dr. Alberto Consolaro

Alameda Octávio Pinheiro Brisolla, 9-75 - Vila Universitária

CEP 17012 - 901 - Bauru - SP

alberto@fob.usp.br 